promoted these effects through the membrane receptor GPER1 located in lipid rafts and that inhibition of lipid rafts and GPER1 suppressed SLE serum-induced skin inflammation and expression of inflammatory molecules.

Conclusions We conclude that oestrogen promotes the development of skin injury induced by SLE serum through the membrane receptor GPER1 and that lipid rafts play an important role in the regulatory effect of GPER1 in SLE skin inflammation.

\section{THE MAJOR ROLES OF MONOCYTES AND THEIR PRODUCT TUMOURTUMOR NECROSIS FACTOR ALPHA IN THE INDUCTION OF SKIN INFLAMMATION TRIGGERED BY INTRADERMAL LUPUS IGG}

${ }^{1} \mathrm{G} \mathrm{Xu},{ }^{2} \mathrm{~L}$ Liu, ${ }^{3} \mathrm{GM}$ Deng. ${ }^{1}$ Nanjing Medical University, Key Laboratory of Antibody Techniques of Ministry of Health, Nanjing, China; ${ }^{2}$ Harvard Medical School, Beth Israel Deaconess Medical Centre, Boston, USA; ${ }^{3}$ Nanjing, China

\subsection{6/lupus-2017-000215.211}

Background and aims Skin injury is the second most common clinical manifestation in patients with systemic lupus erythematosus (SLE), but its pathogenesis has not been thoroughly elucidated.

Methods Based on skin deposition of IgG in SLE, we studied the features and mechanisms of intradermal IgG-induced skin inflammation

Results We found that skin inflammation appeared at 3 hour and peaked at $3 \mathrm{~d}$ after intradermal injection of lupus IgG. This phenomenon was related to the dose of injected IgG but not to systemic disease activity. The severity of skin inflammation induced by lupus IgG was significantly decreased in mice depleted of monocytes and in mice deficient in TNF-a but not in mice lacking mature lymphocytes. Furthermore, lupus IgG promoted the progression of monocyte differentiation to dendritic cells (DCs) and enhanced the expression of TNF- $\alpha$. TNF- $\alpha$ was found to stimulate the IgG-induced maturation of DCs and played a major role in the proliferation and activation of keratinocytes.

Conclusions The results also indicate that the deposition of $\operatorname{IgG}$ in skin exerts an important role in the pathogenesis of skin injury in patients with SLE; therefore, blocking the IgG/ FcR signalling pathway can be a therapeutic target in skin lesions of patients with SLE.

\section{THE ROLE OF IL-1 IN SKIN INFLAMMATION INDUCED BY LUPUS SERUM IGG}

X Li, X Guo, G Xu, GM Deng. Nanjing Medical University, Key Laboratory of Antibody Techniques of Ministry of Health, Nanjing, China

\subsection{6/lupus-2017-000215.212}

Background and aims Systemic lupus erythematosus (SLE) is a seriously chronic autoimmune disease, which is characterised by a large number of autoantibodies and multiple organ damage. Skin lesion is one of the common clinical manifestations of lupus erythematosus, but its pathogenesis is not clear. IL-1 is a proinflammatory cytokine, the aim in this study is to investigate the role of IL-1 in the skin injury in SLE.

Methods We used IL-1 receptor deficient mice and other gene deficient mice to study the role of IL-1 in the lupus serum IgG -induced skin inflammation.
Results We found that the severity of skin lesion in IL-1 receptor deficient mice and caspase-1 deficient mice was reduced compared with that in wild type mice. IL-1 receptor deficiency suppressed the expression of Fc $\gamma R I$ (CD64) and MHC class II (CD74), and increased the level of Fc $\gamma$ RII (CD32) induced by lupus serum. IL-1 receptor deficiency also suppressed the lipid raft clustering and IFN- $\gamma$ in T cells, and reduced IgG internalisation and presentation in macrophage, and decreased expression of MCP-1 and TNFa in monocytes. In addition, TNFa could promote the proliferation of kerationocytes.

Conclusions Our findings indicate that IL-1 plays an important role in skin lesions of lupus erythematosus. This study suggests IL-1 is a therapeutic target in skin lesions of systemic lupus erythematosus.

\section{HEPATIC DEPOSITED IGG MEDIATED LIVER DAMAGE THROUGH KUPFFER/NATURAL KILLER CELLS AND THEIR PRODUCTS}

${ }^{1} \mathrm{X}$ Fang* ${ }^{*}{ }^{1,2} \mathrm{GM}$ Deng. ${ }^{1}$ Nanjing Medical University, Key Lab of Antibody Techniques of Ministry of Health, Nanjing, China; ${ }^{2}$ Harvard Medical School, Beth Israel Deaconess Medical Centre, Boston, USA

\subsection{6/lupus-2017-000215.213}

Background and aims Hepatic disorders are frequent in patients with systemic lupus erythematosus (SLE), yet the aetiology and pathogenesis of liver injury in SLE remains unclear. The present study primarily aimed to understand the cellular and molecular mechanisms involved in the expression of liver damage in SLE.

Methods We analysed clinical and serological characteristics of 404 SLE patients with liver dysfunction, and determined the pathogenesis of liver damage in SLE by using lupus-prone mice and a novel animal model of liver injury induced by lupus serum $\operatorname{IgG}$.

Results $22.5 \%$ of SLE patients have liver dysfunction, and even $38 \%$ of them have lupus-hepatitis. There are a large amount of inflammatory cells around the portal areas of the livers, apoptotic hepatocytes and $\operatorname{IgG}$ deposition in the liver in lupus-prone mice. Liver injury was successfully established by intrahepatic injection of lupus serum IgG. Immune complexes (ICs) stimulated Kupffer cells (KCs) to secrete TNF- $\alpha$ involved in the development of inflammation and apoptosis in liver. IFN- $\gamma$ produced by activated natural killer cells (NKs) directly mediated liver damage and also enhanced the TNF- $\alpha$-mediated apoptotic pathway. The depletion of KCs and NKs abolished apoptosis induced by ICs in liver, suggesting that KCs and NKs have a synergic effect on liver injury.

Conclusions Our findings demonstrated that liver injury was induced by hepatic IgG deposition in SLE and innate immune cells and their products exert an important role in the development of liver injury in SLE. Our results may promote to develop potential therapeutic strategies in prevention and treatment of liver injury in SLE.

\section{RHUPUS SYNDROME : A CASE REPORT AND LITERATURE REVIEW}

A Fikny*, P Faridin. Hasanuddin University, Internal Medicine, Makassar, Indonesia

10.1136/lupus-2017-000215.214 